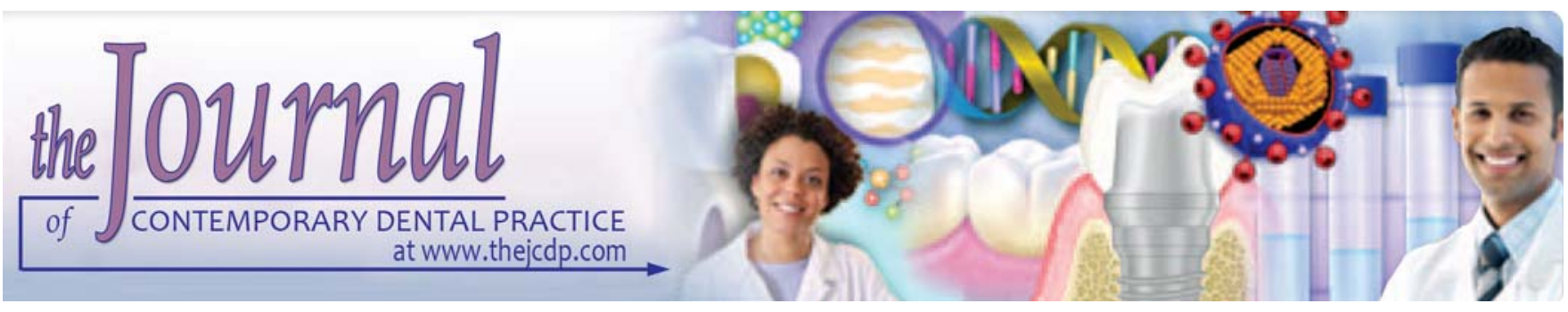

\title{
A Comparative Evaluation of Flexural Strength of Commercially Available Acrylic and Modified Polymethylmethacrylate: An in vitro Study
}

Teerthesh Jain, Naveen S Yadav, Amrita Pandita, SMA Feroz, UK Kartika, Praval Pratap Singh

\section{ABSTRACT}

Aim: The purpose of this study was to evaluate and compare the flexural strength of commercially available acrylic (trevalone) and modified polymethylmethacrylate (PMMA).

Materials and methods: Four groups were tested; Group 1control group regular MMA, group 2-2\% methacrylic acid, $88 \%$ MMA, group 3-16\% methacrylic acid, $84 \%$ MMA group 4$20 \%$ methacrylic acid, $80 \%$ MMA 15 resin specimens of each group were polymerized. After processing, the specimens were subjected for flexural strength testing using three point bending test in a Universal Testing Machine. All data was statistically analyzed with one-way ANOVA, differences within the groups were analyzed by Scheffe's analysis.

Results: As the ratio of incorporated methacrylic acid to PMMA increased, the flexural strength decreased. Analysis of data revealed a significant decrease in flexural strength of specimens ( $p<0.000$ ) after incorporation of 12\%, 16\%, 20\% methacrylic acid to heat polymerized acrylic resin, when compared with the control group. Lowest flexural strength was observed with specimens containing $20 \%$ methacrylic acid and highest flexural strength was observed with specimens containing conventional monomer without methacrylic acid.

Conclusion: It was observed that as the concentration of methacrylic acid in heat polymerized acrylic resin increases, the flexural strength decreases. Lowest flexural strength was observed with specimens containing $20 \%$ methacrylic acid and highest flexural strength was observed with specimens containing conventional monomer without methacrylic acid.

Clinical significance: The major advantages of addition of methacrylic acid to polymethylmethacrylate could be for the elderly people with restricted manual dexterity or cognitive disturbances, especially for patients who do not follow an adequate denture cleansing protocol and diabetic patients who are more susceptible for denture stomatitis.

Keywords: P olymethylmethacrylate, Methacrylic acid, Flexural strength.

How to cite this article: J ain T, Yadav NS, Pandita A, Feroz SMA, Kartika UK, Singh PP. A Comparative Evaluation of Flexural Strength of Commercially Available Acrylic and Modified
Polymethylmethacrylate: An in vitro Study. J Contemp Dent Pract 2013;14(1):80-83.

\section{Source of support: Nil}

Conflict of interest: None declared

\section{INTRODUCTION}

Synthetic acrylic resin have a long, clinically proven history of use for dentures since they exhibit adequate physical, mechanical and esthetic properties. But, they are susceptible to microbial adhesion, leading to denture stomatitis. ${ }^{1}$ Denture stomatitis is frequent among denture wearers and varies widely, reported prevalence range from 10 to $75 \%{ }^{2}$

Surface characteristics resulting from chemistry are significant in the initial adherence of the Candida al bicans to the denture. ${ }^{3,4}$

Physical and mechanical properties of polymers are crucial in achieving clinical success and longevity of complete dentures fabricated. Important physical properties include the following: Compressive and tensile strengths, hardness, thermal characteristics, polymerization shrinkage; solubility and dimensional accuracy. ${ }^{5}$

The flexural failure of denture base materials is considered the primary mode of clinical failure. Smith has suggested that repeated flexing from chewing ultimately fatigues many dentures in the mouth. ${ }^{6}$ However, there is sparse literature regarding the flexural strength after incorporating methacrylic acid in PMMA denture base resins.

Physical strength of these PM M A resins modified with methacrylic acid must be investigated in order to be accepted for daily clinical use. ${ }^{3}$

Hence, the purpose of this study is to evaluate and compare the flexural strength of commercially available acrylic (Trevalon) and mPM M A. 
A Comparative Evaluation of Flexural Strength of Commercially Available Acrylic and Modified Polymethylmethacrylate

\section{MATERIALS AND METHODS}

A total number of 60 specimens were fabricated. The specimens were divided into four experimental groups, consisting 15 specimens in each group. The groups were as follows:

Group 1: Control group pure methylmethacrylate (M M A) Group 2: 12\% methacrylic acid: 88\% M M A

Group 3: $16 \%$ methacrylic acid: $84 \%$ M M A

Group 4: 20\% methacrylic acid: $80 \%$ M M A

The specimens were fabricated according to ADA specification no. 12 (measuring $65 \times 10 \times 2.5 \mathrm{~mm}$ ), using chemicals as indicated in Table 1 . Resins were mixed according to the manufacturer recommended polymer and monomer ratio ( $3: 1$ by volume). Polymerization of the specimens was carried out using a standard processing cycle. The specimens were rinsed and stored in sterile distilled water for 24 hours before use.

Table 1: Materials used, trade name and manufacturer

\begin{tabular}{lll}
\hline Material & Trade name & Material type \\
\hline Denture base resin & Trevalon & $\begin{array}{l}\text { Heat cure denture base } \\
\text { polymer and monomer }\end{array}$ \\
Methacrylic acid & $\begin{array}{l}\text { Rolex laboratory } \\
\text { reagent }\end{array}$ & Liquid \\
\hline
\end{tabular}

\section{Mechanical Testing}

Utilizing a 3-point flexural test, the samples were mounted in a calibrated Instron U niversal Testing M achine (Instron Corp., Canton, M A (Fig. 1). The peak load (fracture load) was recorded in chart recorder.

The peak load is converted to flexural strength by the formula:

$$
\begin{aligned}
& S=3 P L / 2 b d^{2} \\
& S=\text { Flexural strength }\left(\mathrm{N} / \mathrm{mm}^{2}\right) \\
& P=L \text { oad at fracture } \\
& L=\text { Distance between jig supports }
\end{aligned}
$$

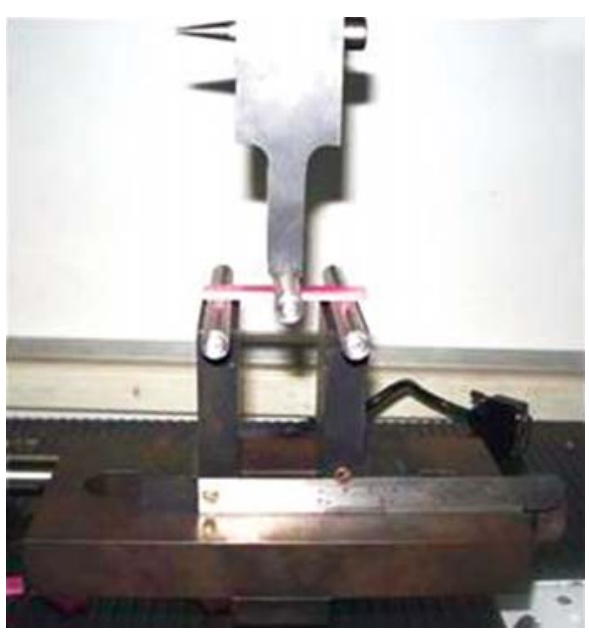

Fig.1: Flexural strength testing using Universal Testing Machine

$$
\begin{aligned}
& b=\text { Specimen width } \\
& d=\text { Specimen thickness }
\end{aligned}
$$

\section{Statistical Analysis}

The mean, median and mode were calculated for each experimental group. Distribution curves were analyzed for normality and one-way analysis of variance (A NOVA) and Scheffe post hoc test were used to compare means between groups.

\section{RESULTS}

A representation of the difference in mean flexural strength is shown in Table 2. The $12 \%$ mPM M A group showed the highest mean flexural strength required. A comparison of mean flexural strength revealed significant difference between the control and the $12 \%$ methacrylic group. Oneway ANOVA analysis demonstrated a highly significant difference $(p<0.0001)$ between the control and the test groups (Table 3).

\begin{tabular}{lcccc}
\multicolumn{5}{c}{ Table 2: Descriptive statistics } \\
\hline Groups & A & B & C & D \\
\hline Mean & 103.8000 & 90.1000 & 85.7000 & 81.4000 \\
Std.deviation & 1.15416 & 1.18040 & 1.26582 & 1.32492 \\
\hline
\end{tabular}

Group A: Control group; Group B: $12 \%$ methacrylic acid; Group C: $16 \%$ methacrylic acid; Group D: $20 \%$ methacrylic acid

\begin{tabular}{lrcc}
\multicolumn{4}{c}{ Table 3: One-way ANOVA-Group } \\
\hline & Df & F & Sig. \\
\hline Between groups & 3 & 578.392 & 0.000 \\
Within groups & 54 & & \\
\hline Total & 57 & & \\
\hline
\end{tabular}

The graph (Fig. 2) depicts mean flexural strength values of all the four groups. The highest flexural strength value is seen with group $A$ and the lowest flexural strength value is seen with group D. The flexural strength value significantly decreases as the concentration of methacrylic acid increase.

As the ratio of methacrylic acid M MA increased, the flexural strength decreased. The $20 \%$ mPMMA group showed a decrease in flexural strength that was statistically significant compared to the $12 \%$ mPM MA group ( $p<$ 0.0001 ). A ll values were well above the minimum value $65 \mathrm{MPa}$ set forth by the ADA specification no.12.

\section{DISCUSSION}

A crylic resins is the most widely used denture base material because of its superior esthetics, favorable working characteristics, processing ease, accurate fit, stability in the oral environment and use with inexpensive equipment. B ut 


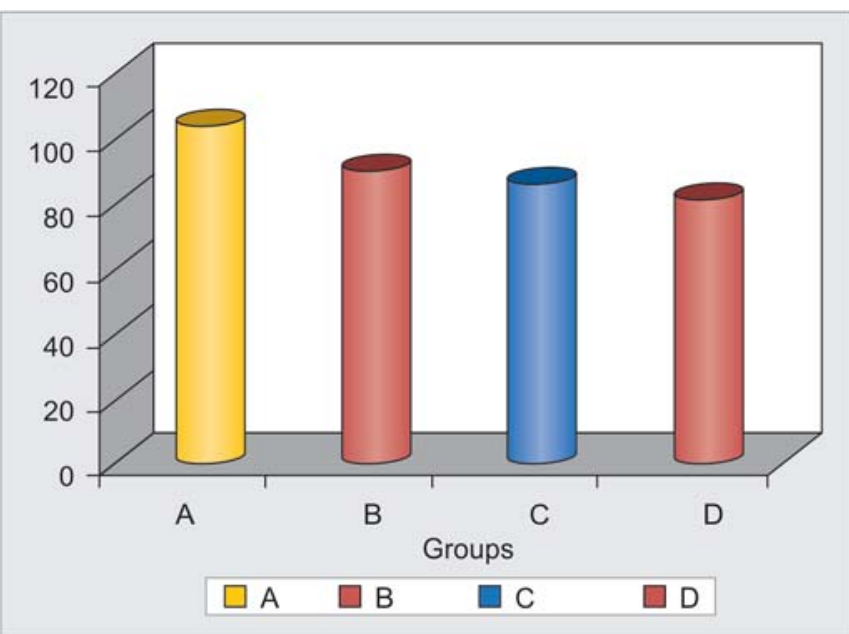

Fig. 2: The mean flexural strength values of all groups

one of the disadvantages of acrylic resin is that, the tissue surface of denture base is susceptible to adherence of Candida albicansand, hence, serves as an effective reservoir of microorganisms. ${ }^{7}$

Chemical disinfectants as chlorine dioxide, sodium hypochlorite, $4 \%$ chlorhexidine and glutaraldehyde are a recommended method to sanitise the prosthesis. However, chemical disinfection may present disadvantages like denture staining and brownish discoloration of the teeth and even the acrylic denture base. ${ }^{8}$

Studies have shown that antifungal drug resistance hinders the successful clinical treatment of candidiasis in denture wearers. Therefore, it is prudent to evaluate alternative materials which may hinder $C$ andida adherence. ${ }^{9}$

Fungal cells are enclosed by cell walls, forms first barrier in direct contact with the biomaterial surfaces. Cell wall provide mechanical and chemical protection. The basic constituents of fungal cell walls are $\beta-$-I, 3-glucans, $\beta-1$, 6 -glucans, chitin and mannoproteins. $\beta$-glucans account for 50 to $60 \%$ of the cell wall by weight; mannoproteins, $40 \%$. These cell wall constituents form a layered structure with mannoproteins mainly on the outside and the glucan layers on the inside.

Therefore, we can speculate that mannoproteins are responsible for the initial interaction with the polymeric surfaces owing to their position. ${ }^{4}$ Phosphorylation of mannosyl side chains contributes to its anionic surface charge in yeasts. It has been demonstrated that outer chain mannosylation is correlated with cell surface hydrophobicity.

Candidal adherence to denture base resin can be attributed to the substantial difference in the protein composition of the acquired enamel pellicle in comparison to denture pellicle. The acquired enamel pellicle contains antimicrobial peptides like histatins along with other constituents, such as IgA, amylase, salivary statherins and mucins. In contrast, the denture pellicle lacks salivary statherins and histatin. The absence of these important salivary defence molecules on the denture base has been attributed to the lack of anionic charge in polymethyl methacrylate (PM M A) polymers. Thus, this lack of surface charge may be responsible for the decreased protective function of the acquired pellicle on the denture. ${ }^{9}$

The addition of methacrylic acid made the new polymer (mPMMA) more hydrophilic and showed a significant correlation between the amount of methacrylic acid in the polymer and decrease in adhesion of C. albicans. ${ }^{4}$ These positive findings made the new surface-modified denture resins attractive for future dental applications.

Although these methods have been effective in reducing the adhesion of $\mathrm{C}$. albicans to the acrylic surfaces, there are concerns regarding the physical properties of these modified polymers. Fractures may occur in use because of its unsatisfactory transverse strength, impact strength or fatigue resistance. ${ }^{10}$

Zappini et al noted that flexural strength is important because it reflects the rigidity of material. For this reason, flexural bend test was selected as it was the most relevant to clinical conditions of the masticatory load to evaluate the strength of acrylic resins. ${ }^{11}$

The ability of Candida albicans to adhere to polymeric surface has been correlated with attractive hydrophobic and repulsive electrostatic forces. ${ }^{4}$

Studies show that negatively charged denture base materials can prevent adhesion of $\mathrm{C}$. albicans and reduce the development of denture-induced stomatitis due to electrostatic interactions. ${ }^{4}$

Thus, there exists a repulsive electric force betw een the negative charge of the MPMMA from the carboxylate groups and the charges or hydrophobic nature of the cell wall of $\mathrm{C}$. albicans due to the mannoproteins.

In the present study, prepolymerizing or mixing two different types of monomers, methacrylate and methacrylic acid produced a copolymer. Methacrylic acid is a small molecule with a free carboxyl group providing a negative charge at physiologic pH. Stearic interactions can be postulated as the free carboxyl group altering the spatial structure of the new polymer, thereby affecting its physical properties. By creating an ionic molecule, stearic hindrance probably causes repelling forces within the resin material. The influence of these internal forces becomes apparent, when a material is subjected to physical testing.

A statistically significant decrease of flexural strength in comparison to the control groups was observed as increasing the methacrylic acid content of the resin samples, which probably resulted from an increase in internal repulsive forces which is consistent with the findings of previous investigations. 
A Comparative Evaluation of Flexural Strength of Commercially Available Acrylic and Modified Polymethylmethacrylate

However, it is important to realise that the flexural strength values obtained for the samples comply with the minimum value ( $65 \mathrm{MPa}$ ) set forth by the ADA specification no.12 and it may have no clinical relevance. ${ }^{12}$

The present study has its limitations because it was done under laboratory conditions. The specimens prepared in this study were not of denture base configuration.

Further modifications may be needed for the modified resins to improveits physical properties while still exhibiting its beneficial antifungal characteristics. A range of methods have been reported for improving the strength of resins through chemical modification of PMMA and through incorporation of fibers, such as carbon, glass, aramid and polyethylene. ${ }^{7,13,14}$

\section{CONCLUSION}

The mechanical tests suggest that incorporation of methacrylic acid decreases the flexural strength of heat polymerized acrylic resins. Nevertheless, the addition of methacrylic acid to polymethylmethacrylate may be effective against microorganisms and, therefore, its impact on mechanical properties may be less significant than the potential benefits.

\section{CLINICAL SIGNIFICANCE}

The major advantages of addition of methacrylic acid to polymethylmethacrylate could be for the elderly people with restricted manual dexterity or cognitive disturbances, especially for patients who do not follow an adequate denture cleansing protocol, and diabetic patients who are more susceptible for denture stomatitis.

\section{REFERENCES}

1. Dhir G, Berzins DW, Dhuru V B, Rajperiathamby A. Physical properties of denture base resins potentially resistant to $C$ andida adhesion. J ournal of Prosthodontics 2007;16(6):465-72.

2. J ean $B$, J acynthe $S$, J ean PG, L ouis DK, Sylvie LA. Reassessing the presence of $C$ andida albicans in denture-related stomatitis, Oral Surg Oral M ed Oral Pathol Oral Radiol Endod 2003;95: 51-59.

3. Sang EP, Ryan B, Srinivas M S, Hans-Peter W. Candida al bicans adherence to surface-modified denture base surface. J ournal of Prosthodontics 2008; 17:365-69.

4. Park SE, Periathamby AR, L oza JC. Effect of surface-charged poly (methylmethacrylate) on the adhesion of Candida al bicans. Journal of Prosthodontics 2003;12(4):249-54.

5. U memoto K, Kurata S. B asic study of a new denture base resin applying hydrophobic methacrylate monomer. D ental M aterials Journal 1997;16(1): 21-30.

6. Chitchumnong P, Brooks SC, Stafford. Comparison of three and four-point flexural strength testing of denture base polymers. Dental M aterials 1989;5:2-5.
7. J ohn J, Shivaputtrappa A, Gangadhar, Shah I. Flexural strength of heat polymerized PM M A denture resin reinforced with glass, aramid or nylon fibers. Journal of Prosthetic Dentistry 2001;86:429-49.

8. Hamoudaa IM, A hmedb SA. Effect of microwave disinfection on mechanical properties of denture base acrylic resin. J ournal of Mechanical Behaviour of Medical M aterials 2010 Oct;3(7):480-87.

9. Puri G, D avid W, Dhuru V B , Periathamby A , Sameer K, Gunjan. Effect of phosphate group addition on the properties of denture base resins, J ournal of Prosthetic D entistry 2008;100:302-08.

10. U zum G, N urhersek, Teomantiancer. Effect of five woven fibre reinforcement on the impact and transverse strength of denture base resin. J ournal of Prosthetic Dentistry 1999;81:616-20.

11. K aracaer 0 , Polat TN, Tezvergil A, Lassila VJ, V allittu PK. The effect of length and concentration of glass fibers on the mechanical properties of an injection and compression-molded denture base polymer. Journal of Prosthetics Dentistry 2003; 90:385-93.

12. A manda, Letícia, Nathália. Effect of denture cleansers on physical properties of heat-polymerized acrylic resin. J ournal of Prosthodontic Research 2010;54:78-83.

13. Rodford R. The development of high impact strength denturebase materials. J ournal of Dentistry 1986;14(5):214-17.

14. Vallittu PK. Dimensional accuracy and stability of polymethylmethacrylate reinforced with metal wire or with continuous glass fiber. The Journal of Prosthetic Dentistry $1996 ; 75(6): 617-21$.

\section{ABOUT THE AUTHORS}

\section{Teerthesh Jain (Corresponding Author)}

Senior Lecturer, Department of Prosthodontics, People's Dental A cademy, B hopal, M adhya Pradesh, India, Phone: 9713392289 e-mail: dr.teerthesh.jain@gmail.com

\section{Naveen S Yadav}

Professor, Department of Prosthodontics, People's D ental A cademy Bhopal, M adhya Pradesh, India

\section{Amrita Pandita}

Senior Lecturer, Department of Prosthodontics, Saraswati-D hanwantari Dental College and Hospital, Parbhani, M aharashtra, India

\section{SMA Feroz}

Senior L ecturer, Department of Prosthodontics, People's Dental A cademy, B hopal, M adhya Pradesh, India

\section{UK Kartika}

Postgraduate Student, Department of Prosthodontics, People's Dental A cademy, B hopal, M adhya Pradesh, India

\section{Praval Pratap Singh}

Postgraduate Student, Department of Prosthodontics, People's Dental A cademy, B hopal, M adhya Pradesh, India 